\title{
区域规划环境评价的空间尺度效应 对上海高桥镇和浦东新区的案例研究
}

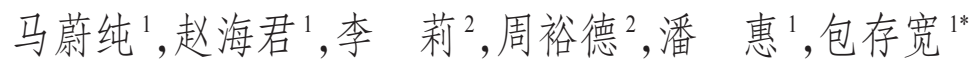 \\ (1. 复旦大学环境科学与工程系, 上海 200433; 2. 上海市环境科学研究院, 上海 200233)
}

\begin{abstract}
摘 要: 区域规划环境评价的空间特性决定其对空间尺度的敏感性。本文从理论层面对区域规划环评中空间尺度 与基础数据的关系、空间尺度与显著环境因子识别以及评价指标体系的关系、空间尺度与大气环境影响预测的关 系进行了初步探讨。并以“上海高桥镇区域规划环境影响评价”(2005年)和“上海浦东新区国民经济与社会发展规 划战略环境评价” (2010年)为例, 通过对比方法, 进一步说明空间尺度与显著环境因子识别和评价指标体系以及与 环境影响预测的关系。结果表明: 基础数据收集、汇总是区域规划环评中极为重要的环节, 由于不同类型的基础数 据表现出不同的空间特征, 需要根据评价区域的实际和评价要求, 合理选择数据所涵盖的空间范围和空间分辨 率。区域规划环境评价应根据本身的层次、涉及范围, 篮选出合理的显著环境因子, 并建立相应的评价指标体系。 大尺度上环境空气质量预测评价的结果侧重于总体描述、解释污染物散布的宏观空间格局和趋势, 而在小尺度上 则更有利于揭示局地精细化的污染扩散规律。
\end{abstract}

关 键 词: 区域规划环境评价; 空间尺度; 基础数据; 显著环境因子; 评价指标体系; 大气环境影响预测

1 引言

区域规划环境评价属于规划层次的战略环境 评价 (Strategic Environmental Assessment, SEA) (Thérivel et al, 1992; 赵倩等, 2010), 是力图从源头 和宏观上预防、减缓人类活动对环境的不利影响, 实现可持续发展的有效手段(Thérivel et al, 1996)。 然而, 目前无论从国内还是国际上来看, 包括区域 规划环评在内的 SEA 基本理论、技术方法都处于探 索阶段,其中一个长期被忽视的重要问题就是预测 评价的尺度效应(João, 2002, 2007a)。

研究表明, 尺度问题是科学界一个十分重要的 问题(Tate et al, 2001; Goodchild, 2011), 是决定观察 事物的视窗大小(Goodchild et al,1997)。尺度又是 最基本最容易混淆和模糊的概念 (Peterson et al, 1998)。一般来说, 尺度包括空间和时间两个方面
(Gontier, 2007; João, 2007b; Partidário，2007)。其 中, 空间尺度包括范围(Extent)和粒度(即空间分辨 率或最小单位, Rsolution)(João, 2007b); 时间尺度包 括代际时间尺度(the Generational Time Scale)和决 策时间尺度 (the Decisional Time Scale) (Partidário, 2007)。近年来, 空间尺度的相关研究在国内日益 受到重视。如钟中等(2007)利用 740 个测站 45 年的 降水资料研究了中国降水年际和年代际变率对空 间尺度的敏感性。游丽平等(2008)选取 8 个常用的 景观指数, 以厦门岛的土地利用格局为例, 分析了 景观指数的空间尺度效应。赵冠伟等(2011)研究了 基于马尔科夫过程的元胞自动机模型模拟土地利 用变化时的空间尺度敏感性特征,结果表明,元胞 尺度的选择会明显影响模拟结果。周杰文等(2011) 选取省、市和县三级空间尺度, 研究了中国中部地 区经济差异的空间尺度效应,并指出“进行区域经

收稿日期: 2014-11; 修订日期: 2015-02。

基金项目: 国家自然科学基金项目(71173049)。

作者简介: 马蔚纯(1969-), 男,上海人,副教授,主要从事战略环境评价、大气模式与环境信息系统方向的研究,

E-mail: wcma@fudan.edu.cn。

通讯作者:包存宽(1970-), 男,山东嘉祥县人,教授,主要从事战略环境评价、环境规划与管理、生态城市等方向的研究,

E-mail: baock@fudan.edu.cn。

引用格式: 马蔚纯, 赵海君, 李莉, 等. 2015. 区域规划环境评价的空间尺度效应: 对上海高桥镇和浦东新区的案例研究 [J]. 地理科学进展, 34(6): 739-748. [Ma W C, Zhao H J, Li L, et al. 2015. Spatial scale of regional plan environmental impact assessment: the Gaoqiao Town and Pudong New District cases in Shanghai, China[J]. Progress in Geography, 34(6): 739-748.]. DOI: 10.18306/dlkxjz.2015.06.009 
济差异分析时应尽量选择较小空间尺度”。徐芝英 等(2012)研究了不同空间尺度转换中数据的精度问 题。李军等(2012)采用地形调节统计模型, 研究了 4 种不同空间分辨率的 DEM 对山区气温空间分布模 拟的影响,结果显示: “微观地形因子(坡度和坡向) 随空间分辨率的变化产生显著变化, 明显影响气温 空间分布”。上述研究均表明, 空间相关的模型、方 法具有明显的空间尺度敏感性, 不同空间尺度对研 究结果有着显著影响。

“SEA 中的尺度问题不仅是一个技术问题, 更 重要的是涉及所需回答和阐述的重要问题, 因此, 是 SEA 的核心要素”(João, 2007a)。在环境评价中, 尺度问题的关键是尺度效应: 即研究不同的尺度如 何影响 SEA 的结果(João, 2007b)。尽管尺度问题在 其他许多学科中已有较多研究, 但却在 2002 年, 尺 度效应研究才首次进人环境评价领域(João, 2002), 因而该问题还远未得到深人研究(João, 2007a)。

在规划 SEA 中, 区域规划环境评价是最具代表 性的类型之一; 由于其空间特性决定了对空间尺度 的敏感性。本文将探讨区域规划环境评价空间尺 度效应的若干问题, 以期对进一步完善区域规划 SEA 的理论框架, 丰富其理论体系提供借鉴和参 考,并为指导区域规划环评的实践提供技术支持。

\section{2 国内外研究进展}

近年来, 在区域规划环境评价的理论研究和实 践中, 尺度问题日益引起重视。Finnveden 等(2003) 指出, 在 SEA 的系统分析中存在几种边界: 人类活 动的地理空间和时间边界(Geographical (and Time) Boundaries for the Activity); 污染排放和资源消耗的 地理空间和时间边界 (Geographical (and Time) Boundaries for Emissions and Use of Resources); 环 境影响的地理空间和时间尺度(Geographical (and Time) Boundaries for Impacts)。在技术层面, 预测 和评价的尺度涉及基础数据库的建立、显著环境因 素识别与评价指标体系、预测评价方法等; 在辅助 决策层面, 直接或间接影响对问题的理解和定义 (Partidário, 2007) 以及评价结论。Gontier(2007)研 究指出, 空间尺度对预测结果和评价结论具有重要 影响。从技术上讲, 在 SEA 中必须选择合适的尺度 进行环境影响预测和评价; 讨论和选择一个特定的 预测和评价尺度具有决定性意义, 尤其是对于累积 影响评价而言。João(2002)通过 GIS 空间叠置分析
和专家咨询两个方法评估道路交通建设项目环境 影响评价中的尺度效应, 结果表明, 尺度变化和尺 度的选择会极大地影响环境影响评价的结论, 亦即 是环评的结果具有尺度依赖性; João 还指出, 尺度 效应在 SEA 中更具有重要价值。Partidário(2007)也 研究指出, 环境评价体现出明显的多尺度性, 并在 SEA 层次体现得更加明显。中国学者李朝晖(2009) 也指出, “空间尺度的选择, 特别是评价范围的选择 对于评价结果有较大影响”。

在 SEA 中, 尺度和数据是相互依存的 (Partidário, 2007)。在一定尺度上相应的数据收集整合 是一个具有挑战性的问题: 由于 SEA 所具有的宏观 性, 因此, 既不宜陷人过于详细的数据中, 又必须收 集、整理充分的数据,为开展预测和评价以及决策 提供重要的信息(João, 2007b)。João研究认为,在 SEA 中, 若评价范围过大, 则数据收集整理的难度 和不确定性增加; 反之, 若评价的地理空间范围过 小, 则对于大尺度的生态环境问题难以有效地考虑 和把握(João, 2002)。对于区域规划 SEA 来说, 可能 在某一空间尺度(空间范围和分辨率)上最宜获得数 据, 或者获得的数据可靠性最高(João, 2007b)。

预测和评价的地理空间范围和分辨率直接关 系到所选用的预测模型(黄懿瑜等, 2003)。大区域 尺度的环境评价难以表达局地小尺度详细的环境 影响(João, 2002)。对于空间分辨率来说, 过粗或者 过细的粒度都不利于得出合理、科学的评价结论 (João, 2007b)。João以道路项目环境影响评价为 例, 详细研究了采用不同基础数据、不同比例尺地 形图和不同缓冲区范围对预测评价结果的影响。 结果表明,在不同空间分辨率背景下,拟建道路缓 冲区内受影响的生态环境敏感目标及数量存在显 著差异; 在区域面积、长度估算方面也存在明显差 异。同时还发现, 由于不同空间分辨率导致生态敏 感目标在空间位置上的差异, 从而导致在拟建道路 与生态敏感目标距离的测量上产生不同的结果 (João, 2002)。一般认为, 在 SEA 中, 模式预测和评 价的空间分辨率并非越精细越好, 它需要与规划的 详细程度、深度相匹配, 与数据的可获得性相匹配, 还需要与评价的目标相一致。

\section{3 区域规划环评中空间尺度效应的表 现形式及案例研究}

依据目前中国开展区域规划环评的实践,在地 
理空间上大致可以分为开发区尺度或镇域尺度(几 ～几十 $\mathrm{km}^{2}$ )、区/县域尺度(几十 几百 $\mathrm{km}^{2}$ )、地级市尺 度(几百 几千 $\mathrm{km}^{2}$ )、省域尺度(数万 数十万 $\mathrm{km}^{2}$ ) 和 大区域尺度。对于不同的尺度, 在基础数据库构 建、重点关注的生态环境问题及显著环境因子识 别、评价指标体系的构建以及环境影响预测的精细 化程度等方面均存在显著差异。本文在国内外现 有工作的基础上, 着重研究在区域规划环境评价 中, 空间尺度与基础数据的关系; 并通过两个案例 对比, 研究空间尺度与显著环境因子识别和评价指 标体系的关系、空间尺度与大气环境影响预测的关 系。本文所指 “空间尺度” 是区域规划环评所涉及 的地理空间范围。

\section{1 空间尺度效应的表现形式}

区域规划环评的空间尺度直接影响人们观察 问题的视窗, 不仅涉及基础数据的收集整理和数据 库建设, 而且还涉及显著环境因子的识别、评价指 标体系的建立, 并影响着环境影响预测和评价的结 论。在技术层面, 基础数据库的构建是开展区域规 划环评首先需要考虑的问题。表 1-3 分别列举了与 区域规划环评密切相关的部分自然地理、经济、人 口和环境等数据的空间尺度特征。在自然地理相 关数据中, 地面气象数据、河流断面水文参数都是
点监测数据, 代表一定区域或一定河段范围内的情 况; 而多年平均水资源量/径流总量、径流深度则往 往以一定流域范围作为统计单元。地形地貌则可 以通过数字高程模型(DEM), 以不同的空间分辨率 予以表达。土壤、植被等分布则可以通过不同比例 尺的专题地图表达,一般来说, 比例尺越大, 表达的 精细化程度越高。

对于经济、人口相关数据, 主要以不同层次的 行政区划为统计单元, 最小的尺度单元一般是乡镇 或各类开发区。对于环境相关数据则情况较为复 杂, 大气、水质、噪声监测绝大部分采用点监测或断 面监测, 而监测点位的设置则往往综合考虑了区域 自然生态环境的代表性和行政区划, 某一点位上的 监测数据往往代表一定行政区划范围的总体情 况。污染物排放与空间尺度的关系则更为复杂, 主 要工业和生活污染源以有组织的点状形态排放; 而 居民生活和农业生产活动所造成的污染排放由于 涉及人口、农业生产活动统计, 目前主要以乡镇、区 县为单元估算; 道路交通源在较小尺度范围内, 例 如开发区、乡镇, 往往可表达为线源, 而在更大尺度 上则一般以面源形式处理。

综上分析, 基础数据本身有着明显的尺度相关 性,不同类型的数据有着不同的空间尺度特征。一

表 1 自然地理相关数据

Tab.1 Data related to the geographic environment

\begin{tabular}{lll}
\hline 数据类型 & \multicolumn{1}{c}{ 具体数据 } & \multicolumn{1}{c}{ 空间尺度特征 } \\
\hline 地面气象数据 & 国家基准站观测数据 & 点监测;代表所在行政区域或其中一定范围的区域 \\
& 一般人工气象站观测数据 & 点监测;代表所在行政区域或其中一定范围的区域 \\
& 自动气象站监测数据 & 点监测;代表站点周边一定范围的区域 \\
& 雨量站降雨量数据 & 点监测;代表站点周边一定范围的区域 \\
& 多年平均水资源量/径流总量、径流深度等 & $1-3$ 级河流的流域尺度 \\
水文水资源数据 & 河流断面水文参数:河流流量、流速、水位等 & 点(断面)监测;代表一定长度的河段 \\
& 数字高程模型(DEM) & 空间分辨率: $100 \mathrm{~m}, 500 \mathrm{~m}, 1 \mathrm{~km}, 10 \mathrm{~km}$ 不等 \\
地形地貌数据 & 土壤类型及其空间分布;植被类型及其空间分布 & 依据专题地图比例尺的不同,表示不同的详尽程度 \\
土壤、植被数据 & &
\end{tabular}

表 2 经济、人口相关数据

Tab.2 Economic and population data

\begin{tabular}{|c|c|c|c|}
\hline \multirow{2}{*}{ 数据类型 } & \multirow{2}{*}{ 具体数据 } & \multicolumn{2}{|c|}{ 统计单元(空间分辨率) } \\
\hline & & 最小统计单元 & 不同层次的统计单元 \\
\hline \multirow[t]{3}{*}{ 经济相关数据 } & GDP 及三次产业的结构比例 & 乡镇、各类经济开发区 & 区县;地级市;省 \\
\hline & 工业、农业总产值/增加值、其他行业的产值/增加值 & 乡镇、各类经济开发区 & 区县;地级市;省 \\
\hline & 经济(GDP)增长率 & 乡镇、各类经济开发区 & 区县;地级市;省 \\
\hline \multirow[t]{3}{*}{ 人口相关数据 } & 人口总量、年龄结构、性别结构、在职人口及其从业结构等 & 乡镇 & 区县;地级市;省 \\
\hline & 人口期望寿命 & 乡镇 & 区县;地级市;省 \\
\hline & 人口素质(受教育程度、平均受教育年限、各类教育层次的人口比例) & 乡镇 & 区县;地级市;省 \\
\hline
\end{tabular}


表 3 环境相关数据

Tab.3 Environmental data

\begin{tabular}{|c|c|c|}
\hline 数据类型 & 具体数据 & 空间尺度特征 \\
\hline 环境质量数据 & 大气、水质、噪声监测数据 & $\begin{array}{l}\text { 点监测; 大气监测点代表所在的行政区域或周边一定范围;水质监测点代表河流某一 } \\
\text { 河段范围;噪声监测点代表邻近范围 }\end{array}$ \\
\hline \multirow[t]{3}{*}{ 污染物排放数据 } & 主要工业污染源、生活污染源 & 以有组织的点状形态排放 \\
\hline & 居民生活面源、农业面源污染 & $\begin{array}{l}\text { 居民日常生活排放往往根据乡镇、区县的人口数据估算; 农药化肥流失、畜禽养殖排 } \\
\text { 放、秸秆燃烧等农业面源往往根据有关统计数据以乡镇、区县等单位估算 }\end{array}$ \\
\hline & 交通源污染 & 在各类开发区、街道乡镇、区县尺度可表现为线源, 在更大尺度范围则往往视为面源处理 \\
\hline
\end{tabular}

方面, 它在空间形态上可以点、线、面等多种方式存 在,也可以不同的空间分辨率(不同尺度的网格、乡 镇、区县等)表达; 另一方面, 对于不同尺度的区域 规划环评,对基础数据所涵盖的空间范围和分辨率 也有不同的要求。对于开发区、镇域等小尺度的区 域规划环评,一般要求空间分辨率更高的基础数 据, 而对于大尺度, 尤其是省域以及跨省尺度上的 区域规划环评, 则要求更为宏观尺度上的基础数 据。例如, 同样是道路交通源的大气污染物排放, 在一般开发区或乡镇尺度,应以线源形式表达; 而 在更大的空间尺度上,则往往以网格面源的形式表 达。可见,在开展区域规划环评构建基础数据库 时,应充分考虑: (1)基础数据本身的空间尺度特征; (2)区域规划环评涉及的地理空间范围。在此基础 上, 综合考虑上述两个空间尺度特征和范围, 根据 评价区域的实际和评价要求, 确定合理的数据收集 整理的空间尺度(空间范围和分辨率), 以达到较好 的数据采集效果和统计分析的精度, 尽可能避免由 于空间尺度转换带来的数据误差。

在不同的空间尺度上, 人们所关注的主要生态 环境问题不同, 从而也影响着显著环境因子的识 别。在大尺度上, 区域生态退化、生物多样性、环境 容量、资源环境承载力、污染物分布的宏观特征等 往往引起人们的重视; 而在小尺度上, 污水纳管能 力、特征污染物排放与治理、局地污染物的时空分 布特征、噪声振动等可能成为重要的环境问题。在 不同尺度的区域规划环评中, 所建立的评价指标体 系也有所不同: 在大尺度上, 除了将显著环境因子 纳人指标体系, 还往往需要结合区域社会、经济、能 源、人口, 尤其是产业发展的现状和规划, 建立综合 性的评价指标体系; 而在小尺度上,则往往基于局 地显著环境因子,建立具有局地特征的评价指标。

评价的空间范围与预测模式的选择、污染源调 查和预测方案也有着密切关系。以大气环境影响 预测为例, 在大区域尺度上应选择具有模拟气象要
素时空变化能力的气象模式,并在空气质量预测中 考虑污染物的化学反应; 而在小尺度上, 则往往可 以采用单一气象站的观测数据代表小范围均一的 气象场,采用高斯烟流模式计算污染物浓度的时空 分布。在大区域尺度上,一般根据区域的环境现状 和特征,篮选对区域生态环境有重要影响的污染源 和污染类型, 编制反映区域污染排放特征的排放清 单,并依据区域国民经济和社会发展规划、产业发 展规划等设置不同的情景开展预测; 而在小尺度 上, 可以进行详尽的污染源调查, 并编制精细化的 排放清单, 同时,由于规划内容更为具体, 不确定性 较小,一般可以较为准确地掌握规划实施后排放清 单的动态变化。

对于预测评价的结果, 在大区域尺度范围, 主 要揭示污染物扩散的宏观空间布局以及重点污染 源对主要生态敏感区和环境保护目标的影响; 而在 小尺度上,一方面重在描述和再现局地范围更为细 致的污染物散布规律, 另一方面也更强调识别小范 围内主要污染源和污染类型对局地环境的影响。

\section{2 案例分析}

本文以“上海高桥镇区域规划环境影响评价” (以下简称“高桥镇规划环评”)和“上海浦东新区国 民经济与社会发展规划战略环境评价”(以下简称 “浦东新区战略环评”)为例, 进一步说明空间尺度 与显著环境因子识别和评价指标体系以及与环境 影响预测的关系。

高桥镇位于上海中心城区的东北翼, 浦东新区 的西北隅, 镇域西北傍依黄浦江, 东北濒临长江 口。镇域面积 $38.73 \mathrm{~km}^{2}$, 中心区面积 $6 \mathrm{~km}^{2}$, 是上 海市域城镇体系 “三城七镇”中的一个中心城镇。 在镇东北,建有外高桥港区、电厂和外高桥保税区 (图 1)。浦东新区开发之初的地理范围包括黄浦江 以东、长江口西南、川杨河以北大约 $350 \mathrm{~km}^{2}$ 的地 域。2003 年拓展至川沙新镇、三林镇等区域, 面积 扩至 $569.57 \mathrm{~km}^{2}$ 。浦东新区开发开放以来, 其建成 
区面积已由 $40 \mathrm{~km}^{2}$ (1990 年)拓展到 $200 \mathrm{~km}^{2}(2008$ 年)(图 1)。

“高桥镇规划环评”属于镇域尺度的规划环评， 而 “浦东新区战略环评”属于区/县尺度的规划环 评。在地域上, 高桥镇隶属于浦东新区; 在产业结 构上,浦东新区在发展规划中确定了电子信息、汽 车、石化、钢材、成套设备、生物医药等六大重点支 柱产业, 高桥镇则是新区能源、石化产业和物流的 重要基地。可见,上述两个规划环评分别对应不同 层次和空间尺度, 在地理上和产业结构上又存在着 包含关系,可以作为本文分析的典型案例。

3.2.1 空间尺度与显著环境因子识别和评价指标体 系的建立

上述两个规划环评都是通过专家咨询, 首先分 析区域存在的主要环境问题, 然后采用影响识别矩 阵进行显著环境影响和因子识别。在“高桥镇规划 环评” 中, 主要环境问题为:工业区尤其是重化工企 业与住宅区相邻, 由此而产生的污染问题严重; 路 网特别是外环线及外环隧道的建成, 造成局地大气 和交通噪声污染严重, 居民投诉不断; 环保基础设 施较差 ${ }^{(1)}$ 。

由此确定地表水、大气和道路噪声为评价重 点。显著环境影响因子主要包括: 水体有机耗氧型 污染的 $\mathrm{COD}_{\mathrm{Cr}} 、 \mathrm{BOD}_{5} 、 \mathrm{NH}_{3}-\mathrm{N}$ 、石油类等; 在大气中, 既 包括 $\mathrm{SO}_{2} 、 \mathrm{NO}_{2} 、 \mathrm{PM} 10$ 等常规污染物, 还包括反映化 工区特征的 $\mathrm{H}_{2} \mathrm{~S}$ 、二甲苯、NMHC等特征污染物; 道路 噪声现状评价和预测因子为连续等效 $\mathrm{A}$ 声级 $\mathrm{L}_{\mathrm{eq}}(\mathrm{A})$ 。

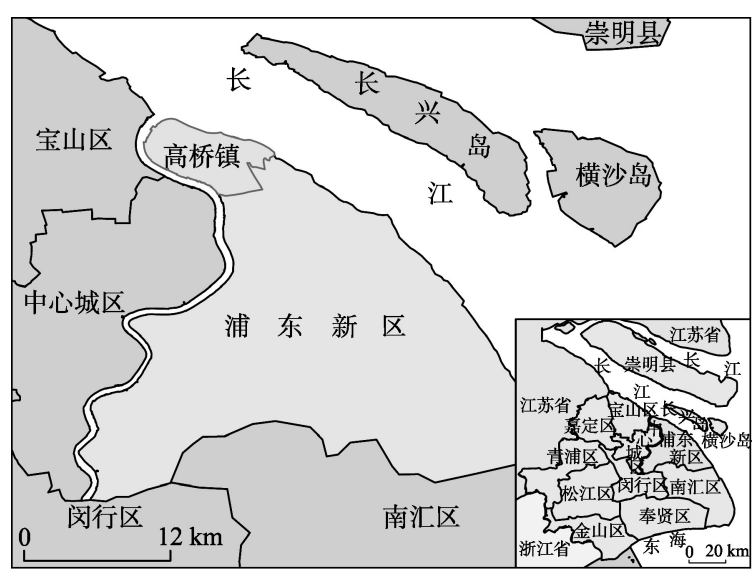

图 1 上海浦东新区及高桥镇的位置

Fig. 1 Location of the Pudong New District and Gaoqiao Town in Shanghai
在“浦东新区战略环评”中, 主要从经济和产业 发展的战略层次分析识别现有和未来的环境问 题。浦东新区在近 20 年的发展中快速完成了工业 化和城市化过程, 形成了以制造业为支撑的产业结 构。但同时,浦东新区也面临着生态环境的巨大压 力,伴随着工业化不断加剧的环境污染问题未来将 面临更为严峻的挑战: 土地资源已近枯竭,能源完 全依靠输人,环境容量趋于饱和。目前浦东新区还 未走出制造业时代, 电子信息产业、石化及精细化 工、生物医药等产业依然是重要的支柱产业，这些 产业的发展规模和空间布局是否合理将对未来的 生态环境产生重要影响; 同时居民生活用能也将呈 现较明显的刚性增长, 使新区节能减排压力增 $大^{(2)}$ 。因此,在区域规划环境评价中需重点考虑的 环境相关因子包括: 综合能耗,水耗,建设用地规模 及土地利用结构及其生态效应, 废水废气产生量, 主要水环境和大气污染物排放量及其环境影响等。

由此可见,不同层次和空间尺度的规划环评所 关注的主要环境问题不同,需识别的显著环境因子 也存在差异。在较高层次和较大的空间尺度上, 区 域规划环评主要关注产业规模、结构及其空间布 局, 与此相应, 能源、水资源消耗、土地利用与建设 规模及其由此而产生的区域性水污染、大气污染以 及生态影响成为显著环境因子。而在开发区或乡 镇尺度上,局地的污染问题成为最重要的环境问 题,在显著环境因子中,除了常规污染物外,还包括 体现局地特征的环境因子。

评价指标与显著环境因子的识别有着直接的 联系。“高桥镇规划环评”中尽管没有专门建立评价 指标体系,但根据显著环境因子识别,对于大气、地 表水和道路噪声, 分别建立了现状评价指标。对于 环境空气和噪声建立了预测评价指标(表 4)。从表 中可见, 在小尺度上除关注常规污染物外, 比较注 重局地特征污染物, 例如体现化工园区特点的 $\mathrm{H}_{2} \mathrm{~S}$ 、 二甲苯、 $\mathrm{NMHC}$ 和丙稀腈等, 而对于人口、经济、能 源消耗、土地利用等相关指标则基本不涉及。

在“浦东新区战略环评”中, 建立了一套较为完 整的评价指标体系(表 5)。从表中可见,随着评价区 域范围的拓展, 对于局地范围的特征性污染指标减 少,而以一般常规性、普适性污染物为主; 同时,增 加了与区域环境容量与污染物排放总量控制相对 应的总量指标。从表中还可以看到,在浦东新区尺

(1)上海市环境科学研究院. 2005. 高桥镇区域建设环境影响评价报告书.

(2)上海市环境科学研究院,复旦大学等, 2010.上海市浦东新区国民经济与社会发展规划战略环境评价研究报告(总报告). 
度的 SEA 中引人了社会、经济、人口、产业发展、能 源消耗、碳排放、土地利用等更为宏观的指标, 以反 映社会经济、人口驱动对生态环境的影响, 也进一 步显示区域的资源环境效率。

综上分析可知, 在不同层次和空间尺度的区域 规划环评中, 所建立的评价指标体系存在显著差 异, 即指标体系也有着尺度相关性和依赖性。在较 高层次和较大尺度上, 区域规划环评是协调经济、 社会发展与环境保护和生态建设, 实现可持续发展 的重要手段, 也是环境保护融人政府综合决策的重 要载体。由于其重点关注的是区域经济、社会、人 口和区域性的生态环境问题, 具有明显的宏观性和 综合性, 因此, 一般需要建立综合性的评价指标体 系。而在开发区、镇域层次和尺度上, 由于其产业 规模、结构、空间布局等的规划已经比较细化和具 体, 更由于与经济社会发展相关的要素已经在更高
层次和更大空间尺度的规划与规划环评中予以考 虑, 因此, 这一层次和尺度的规划环评往往更聚焦 于局地的生态环境问题,所建立的评价指标主要为 狭义的环境预测与评价服务,并不一定构成完整的 指标体系。

\subsection{2 空间尺度与环境影响预测}

通过比较上述两个规划环评中的大气环境影 响预测讨论空间尺度与环境影响预测之间的关系。

(1) 预测模式

“高桥镇规划环评”中大气环境影响预测采用 AERMOD 软件系统, 通过联合频率计算年均浓 度。AERMOD 基于高斯烟流模式,采用空间上均 一的气象场计算污染物的扩散,适用于较小范围的 污染模拟和预测。

“浦东新区战略环评”中大气环境影响预测采 用CALPUFF 软件系统, 网格分辨率 $1 \mathrm{~km} \times 1 \mathrm{~km}$ 。

表 4 高桥镇区域规划环境影响评价中的评价指标

Tab.4 Indicators for the environmental impact assessment of Gaoqiao Town regional plan

\begin{tabular}{|c|c|c|c|}
\hline 评价指标 & 大气环境 & 地表水环境 & 道路噪声 \\
\hline 现状评价指标 & $\begin{array}{l}\mathrm{SO}_{2} 、 \mathrm{NO}_{2} 、 \mathrm{PM} 10 、 \mathrm{H}_{2} \mathrm{~S} \text { 、二甲苯、 } \mathrm{NMHC} \text { 、 } \\
\text { 丙稀腈 }\end{array}$ & $\begin{array}{l}\mathrm{pH} 、 \mathrm{COD}_{\mathrm{Cr}} 、 \mathrm{BOD}_{5} 、 \mathrm{NH}_{3}-\mathrm{N} \text { 、石油类、 } \\
\mathrm{TP} 、 \mathrm{Cr}^{6+} 、 \mathrm{Zn} 、 \mathrm{Cu} 、 \mathrm{~Pb} 、 \mathrm{Ni}\end{array}$ & 连续等效 $\mathrm{A}$ 声级 $\mathrm{L}_{\mathrm{eq}}(\mathrm{A})$ \\
\hline 预测评价指标 & $\mathrm{SO}_{2} 、 \mathrm{NO}_{2} 、 \mathrm{PM} 10 、 \mathrm{CS}_{2} 、 \mathrm{H}_{2} \mathrm{~S} 、 \mathrm{NMHC}$ & - & 连续等效 $\mathrm{A}$ 声级 $\mathrm{L}_{\mathrm{cq}}(\mathrm{A})$ \\
\hline
\end{tabular}

表 5 浦东新区国民经济与社会发展规划战略环境评价中的指标体系

Tab.5 Indicators for the strategic environmental impact assessment of economic and social development plan of Pudong New District

\begin{tabular}{|c|c|c|c|}
\hline 领域层 & 主题层 & 要素层 & 指标层 \\
\hline \multirow[t]{9}{*}{ 社会和经济发展 } & 发展规模 & 经济发展规模 & 人均 GDP \\
\hline & & & 经济密度 \\
\hline & & 社会发展规模 & 人口密度 \\
\hline & & 资源能源消耗 & 万元 GDP 综合能耗 \\
\hline & & & 万元 GDP 用水量 \\
\hline & 经济结构 & 产业结构 & 第三产业占 GDP 的比重 \\
\hline & & & 工业重点行业产值占工业总产值的比重 \\
\hline & & 土地利用结构 & 土地利用强度 \\
\hline & 空间布局 & 工业布局 & 工业企业在工业园区的集中度 \\
\hline \multirow[t]{11}{*}{ 生态环境影响 } & 污染负荷 & 水环境污染负荷 & 化学需氧量排放量 \\
\hline & & & 氨氮排放量 \\
\hline & & 大气环境污染负荷 & $\mathrm{SO}_{2}$ 排放量 \\
\hline & & & $\mathrm{NO}_{\mathrm{x}}$ 排放量 \\
\hline & & & 能源相关碳排放 \\
\hline & 环境质量 & 水环境质量 & 化学需氧量平均浓度 \\
\hline & & & 氨氮平均浓度 \\
\hline & & 大气环境质量 & $\mathrm{SO}_{2}$ 年均浓度 \\
\hline & & & $\mathrm{NO}_{2}$ 年均浓度 \\
\hline & & & PM10年均浓度 \\
\hline & & & 7月份 $\mathrm{O}_{3}$ 小时浓度超标率 \\
\hline
\end{tabular}


通过观测数据(气象观测数据来自 2007 年上海市 6 个地面气象站和 1 个高空站的观测资料)插值获得 地面气象场。CALPUFF 软件系统采用高斯烟团模 式,可采用空间变化的气象场计算污染物的扩散, 较适用于较大空间范围(几百 $\mathrm{km}$ )的污染模拟预测。

(2) 污染源调查、评估与预测方案。

“高桥镇规划环评”中, 通过实地调查列举所有 点源排放口, 进而统计各污染源主要污染物排放 量, 包含燃烧和工艺过程排放。在预测方案编制 中, 依据高桥镇产业导向及发展规划和环保规划, 确定了中远期污染源的具体情况, 落实到污染源关 停并转的详细预测。相对来说, 不确定性较小。

“浦东新区战略环评” 中, 主要考虑点源、流动 源、面源的大气污染排放。点源采用2007年污染源 普查数据, 涵盖了规模以上企业的电站/工业锅炉、 工业窑炉及其相关工艺过程等中高架排放源。流 动源主要为区域内的道路机动车排放, 采用 IVE模 型估算。面源主要包括生活源、道路扬尘及其他无 组织排放源。计算中所需经济总量、人口规模、能 源消耗总量和工业产品产量等数据来自统计年鉴。

在预测方案编制中, 首先根据经济发展和人口 增长等驱动因素, 预测浦东新区各行业能源需求, 再结合新区大气污染控制政策和相关规划, 考虑各 类减排措施, 对各类污染源大气污染物排放进行预 测。对于 2015 年的规划, 根据经济、人口、能源消耗 的发展预期, 设计了 9 种不同的情景方案, 分别预测 主要大气污染物的排放。

(3) 预测结果

图 2 给出了高桥镇远期(2020年)规划实施后本 地源造成的主要污染物浓度空间分布; 图 3 给出了 浦东新区 2015 年规划实施后, 排放量最大的方案本
地源造成的主要污染物浓度分布。图中均显示出 最高值范围、次高值范围及其分布。

从图 2-3 中可见,不同空间范围的排放清单对 预测结果有显著影响。对于 $\mathrm{SO}_{2}$, 高桥镇规划环评 和浦东新区战略环评预测得到的年均浓度基本处 于同一数量级, 从最高值来说, 后者稍大于前者。 后者在预测中, 给出了整个浦东新区范围内 $\mathrm{SO}_{2}$ 年 均浓度的空间分布, 但在高桥镇区域仅给出了浓度 分布的大致规律; 而前者则给出了高桥镇区域内更 为精细的 $\mathrm{SO}_{2}$ 年均浓度空间分布特征, 使评价者更 易识别出小范围内的浓度高值区、次高值区, 并引 导评价者进一步分析造成局地高值的原因。

在 $\mathrm{NO}_{2}$ 和 PM10 预测中, 高桥镇规划环评预测 的年均浓度明显小于浦东新区战略环评中的预测 值, 这很可能与后者在预测中考虑了整个浦东新区 较大尺度的污染源有关。对于 $\mathrm{NO}_{2}$, 在浦东新区 SEA 的预测中, 高桥镇年均浓度大致出现自南向北 递减的趋势, 南部浓度最高, 在 $100 \mu \mathrm{g} / \mathrm{m}^{3}$ 以上, 北部 浓度最低, 在 20 50 $\mu \mathrm{g} / \mathrm{m}^{3}$ 之间; 但在高桥镇规划环 评的预测中, 该镇东北部出现了浓度相对高值区, 在 北部和西北部浓度则较低。对于 PM10, 在浦东新 区 SEA 的预测中, 给出了高桥镇年均浓度的总体分 布格局: 该镇南部和东南部地区的浓度较高, 大于 $50 \mu \mathrm{g} / \mathrm{m}^{3}$; 而在西部、北部地区浓度较低,在 10 50 $\mu \mathrm{g} / \mathrm{m}^{3}$ 之间。但在高桥镇规划环评的预测中, 尽管 浓度值较低, 但给出了更为精细的浓度空间分布, 高值区出现在镇域南部沿黄浦江的区域,并逐步向 北、东北和西北方向递减。这一特征与 $\mathrm{SO}_{2}$ 浓度的 空间分布相似,从而体现出镇域尺度上污染物空间 分布的特征和规律。

综合上述分析可知,不同层次和空间尺度的环
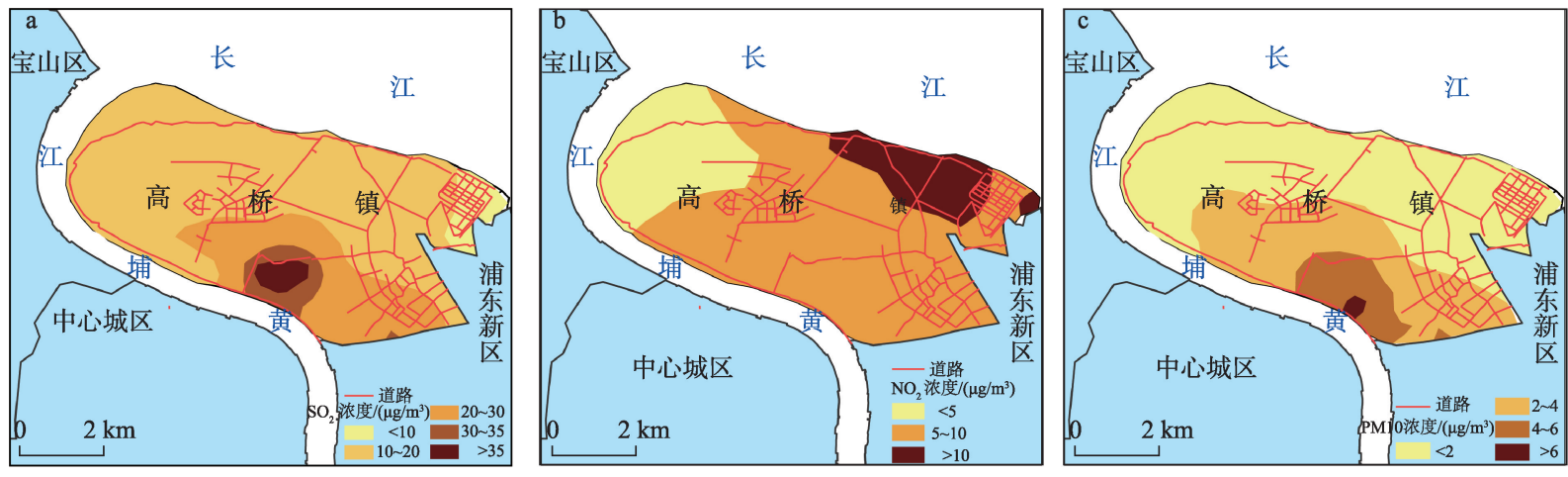

图 2 高桥镇远期(2020年)规划实施后本地源造成的主要污染物浓度分布 (a. $\mathrm{SO}_{2} ;$ b. $\mathrm{NO}_{2}$; c. PM10)

Fig.2 Concentration of main air pollutants caused by local sources after the implementation of the development plan of Gaoqiao Town in year 2020 (a. $\mathrm{SO}_{2}$; b. $\mathrm{NO}_{2}$; c. PM10) 

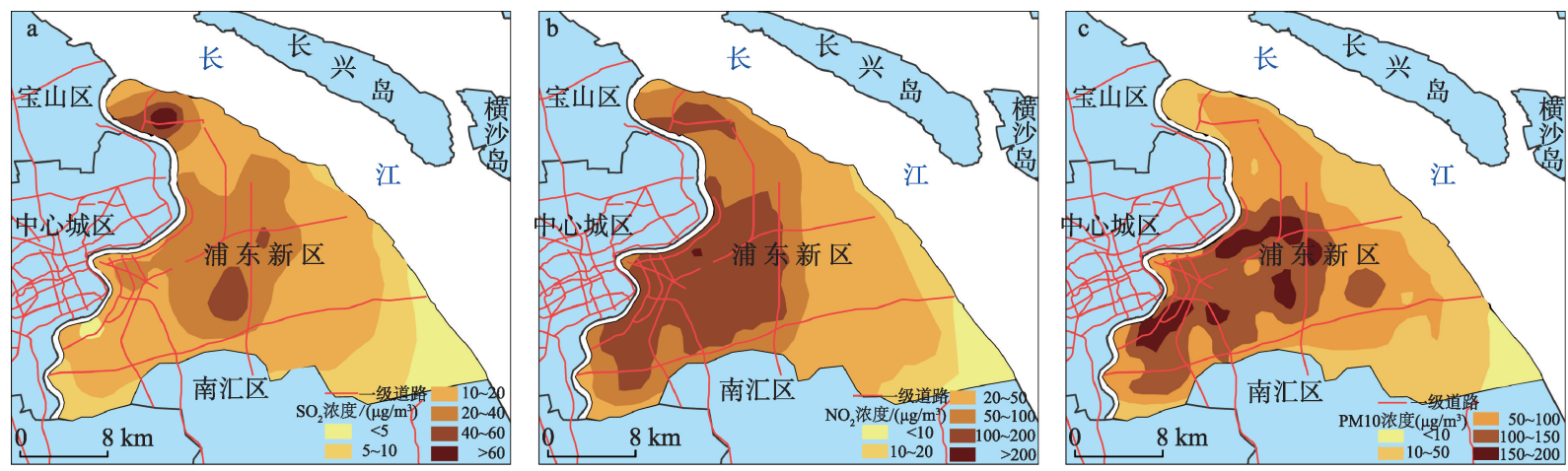

图 3 浦东新区 2015 年规划实施后本地源造成的主要污染物浓度分布(a. $\mathrm{SO}_{2}$; b. $\mathrm{NO}_{2}$; c. PM10)

Fig.3 Concentration of main air pollutants caused by local sources after the implementation of the development plan of Pudong New District in year 2015 (a. $\mathrm{SO}_{2}$; b. $\mathrm{NO}_{2}$; c. PM10)

境影响预测所采用的模型可能不同。模型有其自 身适用的空间范围, 不同尺度的区域规划环评只有 选择与其评价范围相适应的模型才能作出科学合 理地预测。例如, 均一气象场的空气质量模型一般 只适用于小尺度的预测, 而非均一气象场的模型则 可被用于更大尺度, 如省域、甚至跨省范围的空气 质量预测。在开发区和镇域尺度的环境预测中,一 般来说可以采用精细化的污染源排放清单, 未来预 测的不确定性较小; 而在较大尺度的影响预测中, 由于其规划本身的层次较高, 污染源的预测存在较 大的不确定性, 这样, 情景分析就成为环境影响预 测的可选方案, 它不仅能有效降低预测的不确定 性,也可成为大尺度区域规划环评中多方案比选的 手段。从不同空间尺度的预测结果看,在数百 $\mathrm{km}$ 及以上的较大尺度上, 可以展示较大范围污染物空 间分布的宏观特征; 而在开发区或镇域尺度上的模 拟结果则可比较精细地再现小尺度上污染物的空 间分布特征, 尤其是可以反映局地尺度上重要污染 源的环境影响, 识别其对局地污染的浓度贡献。这 将有助于在开发区或镇域层次上制定相应的控制 对策和措施。

\section{4 结论与讨论}

\section{1 结论}

本文结合案例分析, 初步揭示了区域规划环境 评价中空间尺度与基础数据、显著环境因子识别和 评价指标体系建立、预测结果的关系。得到以下主 要结论:

(1) 基础数据的收集、整理是区域规划环评中 极为重要的环节。规划环评属于中观或宏观层次
的战略环评, 是在政府综合决策中考虑生态环境保 护的有效工具, 它涉及自然地理、社会、经济、人口、 能源等各个相关领域, 对基础数据的要求,无论是 数据量和数据类型, 还是复杂性都远远超过项目环 评。从 3.1 节的分析可知, 区域规划环评需要的基 础数据可有点、线、面不同的表现形态,有些数据是 一个点上的监测数据, 有些是一个区域范围内的统 计数据, 还有些数据在不同的空间尺度上可以表现 出不同的空间特征。区域规划环评应根据评价区 域的实际和评价要求,选择合理的基础数据搜集、 汇总的空间范围和合适的空间分辨率。

(2) 区域规划环评中的显著环境因子识别和评 价指标体系针对重点生态环境问题,存在空间尺度 效应。不同层次、不同空间尺度的规划环评所关注 的环境问题不同: 越是高层次、大区域范围的评价, 越是关注宏观、全局性的问题，例如能源、水资源消 耗,产业规模、结构和空间布局, 以及污染物排放总 量等; 而小尺度规划环评则主要关注污染物, 尤其 是局地特征污染物排放及其对环境的影响。可见， 区域规划环评应根据区域规划本身的层次、涉及的 空间范围,抓住相应尺度上最重要和最显著的生态 环境问题开展分析研究,这样才能真正为政府的宏 观决策提供依据。

(3) 空间尺度与预测评价结果也有着重要的联 系。在大区域尺度上的环境影响预测往往涉及大 量的污染源数据和大区域排放清单的编制, 难以对 每一污染排放口进行现场监测与核查, 而重点在于 抓住那些对区域环境有重要影响的污染源和污染 类型。在预测中为降低不确定性, 情景分析是一种 有效的方法。而在小尺度上, 则往往可以列举几乎 所有污染源, 编制精细化的排放清单, 可以更准确 
地掌握规划实施后的污染源及其排放变化。对于 预测评价结果, 大尺度范围的预测往往可从总体上 描述、解释污染物散布的宏观空间格局和趋势, 而 小尺度范围的预测则可揭示局地更为精细的污染 分布规律, 有利于识别出小范围内污染源的影响。 可见, 在区域规划环评的环境影响预测中应根据评 价要求, 选择合适的预测范围, 才能有利于揭示区 域规划实施的环境影响, 得出科学的评价结论, 提 出与规划尺度相适应的污染防控对策。

\section{2 讨论}

本文在国内外文献综述的基础上, 通过理论探 讨和案例分析, 比较全面地阐述了区域规划环评中 的空间尺度效应, 具体包括空间尺度与基础数据的 关系、与显著环境因子识别和评价指标体系的关系 以及与环境影响预测的关系。应当指出的是, 无论 是在国内还是国际上, 关于环境评价空间尺度效应 的研究尚处于起步和探索阶段, 理论探讨和实证研 究都有待于进一步深人。本文也仅就区域规划环 评空间尺度效应中所涉及的若干问题进行了初步 的理论分析, 并基于两个区域规划环评的案例进行 了归纳和总结, 所得出的结论和规律尚是初步的, 其普适性还有待于进一步研究, 尤其是需要更多的 实证研究给予支持和检验。

在未来的研究中, 需要在理论探索和实证研究 两个方面互动推进。在理论层面, 进一步分析规划 环评各环节和要素与空间尺度的关系, 例如本文中 尚未涉及的公众参与、规划的相容性分析与空间尺 度的关系等, 并充分运用相关领域(例如环境预测 模型、公众参与、规划编制等)的研究成果, 开展理 论层面的综合集成分析。环境评价是应用性很强 的研究领域, 没有大量的实践和实证研究也就难以 建立完善的理论体系。因此, 在未来的研究中, 针 对规划环评的空间尺度效应, 需要开展更多的实证 研究, 并与理论探索互动, 一方面为理论研究提供更 多的案例支撑, 另一方面也将对理论研究获得的结论 和规律的可靠性、普适性等进行检验。

\section{参考文献(References)}

黄懿瑜, 马蔚纯, 余琦. 2003. 城市综合交通规划环境评价中 大气环境预测的数学模型 $[\mathrm{J}]$. 上海环境科学, 22(5): 335-338. [Huang Y Y, Ma W C, Yu Q. 2003. Mathematical modeling for air quality prediction in environmental assessment of urban comprehensive transportation planning [J]. Shanghai Environmental Science, 22(5): 335-338.] 李朝晖. 2009. 空间尺度对非污染生态影响评价结果的影
响: 以开发区区域环境影响评价为例 $[\mathrm{J}]$. 海峡科学, (6): 14-15, 25. [Li C H. 2009. Impact of spatial scales on nonpolluting ecological impact evaluation result: using environmental impact assessment of development area as an example[J]. Channel Science, (6): 14-15, 25.]

李军, 黄敬峰, 游松财. 2012. 不同空间尺度 DEM 对山区气 温空间分布模拟的影响: 以浙江省仙居县为例 $[\mathrm{J}]$. 地理 科学, 32(11): 1384-1390. [Li J, Huang J F, You S C. 2012. The different spatial-scaling effect of DEM resolution on the simulation of temperature spatial distribution in mountainous area: a case study in Xianju County, Zhejiang Province[J]. Scientia Geographica Sinica, 32(11): 1384-1390.]

徐芝英, 胡云锋, 刘越, 等. 2012. 空间尺度转换数据精度评 价的准则和方法 [J]. 地理科学进展, 31(12): 1574-1582.

[Xu Z Y, Hu Y F, Liu Y, et al. 2012. A review on the accuracy analysis of spatial scaling data[J]. Progress in Geography, 31(12): 1574-1582.]

游丽平, 林广发, 杨陈照, 等. 2008. 景观指数的空间尺度效 应分析: 以厦门岛土地利用格局为例 $[\mathrm{J}]$. 地球信息科 学, 10(1): 74-79. [You L P, Ling G F, Yang C Z, et al. 2008. The effects of spatial scales on landscape indices: a case study of the land use pattern of Xiamen Island[J]. Geo-information Science, 10(1): 74-79.]

赵冠伟, 陈顷虎, 陈健飞, 等. 2011. CA-Markov模型的空间 尺度敏感性研究 [J]. 地理科学, 31(8): 897-902. [Zhao G W, Chen Y B, Chen J F, et al. 2011. Spatial scale sensitivity of CA-Markov model[J]. Scientia Geographica Sinica, 31(8): 897-902.]

赵倩, 张艳, 余琦, 等. 2010. 基于情景分析的上海浦东外高 桥功能区总体规划大气环境评价 [J]. 复旦学报: 自然科 学版, 49(3): 331-339, 344. [Zhao Q, Zhang Y, Yu Q, et al. 2010. Atmospheric environmental assessment for master plan of Waigaoqiao functional area, Shanghai Pudong New Area[J]. Journal of Fudan University: Natural Science, 49(3): 331-339, 344.]

钟中, 胡轶佳, 闵锦忠. 2007. 中国降水年际和年代际变率对 空间尺度的敏感性 [J]. 地球物理学报, 50(5): 13301336. [Zhong Z, Hu Y J, Min J Z. 2007. Sensitivity of interannual and interdecadal precipitation variability over China to spatial scale[J]. Chinese Journal of Geophysics, 50(5): 1330-1336.]

周杰文, 张璐. 2011. 中部地区经济差异的空间尺度效应分 析 [J]. 地理与地理信息科学, 27(1): 49-52. [Zhou J W, Zhang L. 2011. Spatial scale effects of the regional economic disparities in the central China area[J]. Geography and Geo-Information Science, 27(1): 49-52.]

Finnveden G, Nilsson M, Johansson J, et al. 2003. Strategic environmental assessment methodologies: applications within the energy sector[J]. Environmental Impact Assessment Review, 23: 91-123. 
Gontier M. 2007. Scale issues in the assessment of ecological impacts using a GIS-based habitat model: a case study for the Stockholm region[J]. Environmental Impact Assessment Review, 27: 440-459.

Goodchild M F. 2011. Scale in GIS: an overview[J]. Geomorphology, 130: 5-9.

Goodchild M F, Quattrochi D A. 1997. Introduction: scale, multiscaling, remote sensing, and GIS[M]//Quattrochi D

A, Goodchild M F. Scale in remote sensing and GIS. Boca Raton, FL: Lewis Publishers: 1-11.

João E. 2002. How scale affects environmental impact assessment[J]. Environmental Impact Assessment Review, 22: 289-310.

João E. 2007a. A research agenda for data and scale issues in Strategic Environmental Assessment (SEA) [J]. Environmental Impact Assessment Review, 27: 479-491.

João E. 2007b. The importance of data and scale issues for
Strategic Environmental Assessment (SEA) [J]. Environmental Impact Assessment Review, 27: 361-364.

Partidário M R. 2007. Scales and associated data: What is enough for SEA needs[J]. Environmental Impact Assessment Review, 27: 460-478.

Peterson D L, Parker T V. 1998. Dimension of scale in ecology, resource management and society[M]//Peterson D L, Parker T V. Ecological scale theory and applications. New York: Columbia University Press.

Tate N J, Atkinson P M. 2001. Modelling scale in geographical information science[M]. Chichester, UK: Wiley.

Thérivel R, Partidario M R. 1996. The practice of strategic environmental assessment[M]. London, UK: Earthscan Publication.

Thérivel R, Wilson E, Thomson S, et al. 1992. Strategic environmental assessment[M]. London, UK: Earthscan Publication.

\title{
Spatial scale of regional plan environmental impact assessment: the Gaoqiao Town and Pudong New District cases in Shanghai, China
}

\author{
MA Weichun ${ }^{1}$, ZHAO Haijun ${ }^{1}$, LI Li ${ }^{2}$, ZHOU Yude ${ }^{2}$, PAN Hui ${ }^{1}$, BAO Cunkuan ${ }^{1 *}$ \\ (1. Department of Environmental Science and Engineering, Fudan University, Shanghai 200433, China; \\ 2. Shanghai Academy of Environmental Science, Shanghai 200233, China)
}

\begin{abstract}
The spatial characteristics of Regional Plan Environmental Impact Assessment (RPEIA) determine its sensitivity to spatial scale. Spatial scale of RPEIA is not only a technical issue, but also related to the important questions that RPEIA needs to answer and elaborate. This article discusses the relationship between spatial scale and basic data, the screening of significant environmental factors, and evaluation indicators, as well as the relationship between spatial scale and prediction of atmospheric environmental impact. It then examines two cases: the RPEIA of Gaoqiao Town (2005) and the Strategic Environmental Impact Assessment of the economic and social development plan of Pudong New District, Shanghai (2010) for further elaboration. This article concludes that data collection and integration is a very important step in RPEIA. Different types of basic data posses different spatial characteristics, their spatial scope and resolution should be determined according to the requirements of the RPEIA and regional conditions. The main environmental concerns vary considerably at different spatial scales and they affect the selection of significant environmental factors directly, which in turn affects the corresponding evaluation indicator system. This article also indicates that atmospheric environmental impact prediction and evaluation at large spatial scale focus on the macroscopic description, revealing the general spatial patterns and trends of the spread of air pollutants, while at the local scale is more conducive to explain localized pollution patterns. Emission inventory also emphasizes different spatial characteristics for prediction and evaluation at different scales.
\end{abstract}

Key words: regional plan environmental impact assessment (RPEIA); spatial scale; basic data; significant environmental factors; evaluation indicator system; prediction of atmospheric environmental impact 